\title{
Comment
}

\section{Reply to Comment "Animal Welfare and Ethics in the Collection of Fetal Blood for the Production of Fetal Bovine Serum"}

\author{
Tilo Weber', Frigga Wirths', Nina Brakebusch ${ }^{1}$ and Jan van der Valk ${ }^{2}$ \\ ${ }^{1}$ Animal Welfare Academy of the German Animal Welfare Federation, Neubiberg, Germany; ${ }^{2}$ The 3 Rs-Centre, Utrecht University, Utrecht, \\ The Netherlands
}

doi:10.14573/altex.2103191

For decades, fetal bovine serum (FBS) continues to be a controversially discussed cell culture supplement consisting of animal welfare, ethical, regulatory, health, legal and scientific issues regarding its use, production and distribution (Hodgson, 1991; Jochems et al., 2002; Gstraunthaler et al., 2013; van der Valk et al., 2018). In the meeting report on our 2020 online symposium "Replacing Fetal Bovine Serum (FBS) in Research and Testing", we invited the serum-producing industry to discuss and find a common solution for the animal welfare issues and the development of animal-friendly medium supplements (Weber and Wagner, 2021). We therefore welcome the Comment of Versteegen et al. (2021) and would like to respond and help to further clarify common misconceptions on FBS.

\section{Moral obligations}

Versteegen et al. (2021) begin with a claim that it is a "moral obligation to not only consider the welfare of animals within the food chain but also to maximize the potential of these animals". We disagree, as it should be a moral obligation to maximize the welfare of the animals that are used for human purposes, but not their exploitable potential. It follows that we should first reduce and ultimately replace potential causes for animal suffering.

Furthermore, the term "moral obligation" misses the point when considering the suffering of animals. Morals are variable, fluent concepts of the idea of what is right and wrong. They differ between people, cultural context and time, whereas ethics describe an (academically justified) concept of guiding principles for human conduct. In terms of FBS, it is not about what someone thinks is right or wrong, but about including animal welfare issues into our considerations in general. Animal welfare legislations all over the world testify that animals' suffering matters if there is any good reason to claim that they are suffering.

As we will point out in this rebuttal, animal welfare issues in FBS production are pressing, as there is potential for suffering both for the involved dam as well as for the fetus.

\section{Animal sentience}

Another claim made by Versteegen et al. (2021) is that fetuses are neither capable of perceiving pain while still in utero nor are they alive during blood collection, consequently denying any need for a certified killing method or even anesthesia before cardiac puncture of the fetus. However, the case is not as clear as presented.

The duration of pregnancy is similar in cattle and humans. Sensation of pain is postulated for human fetuses, and therefore anesthesia of the human fetus during prenatal surgery is common practice (Bellieni, 2019). For humans, it is suggested that EEG activity in newborn infants and fetuses barely differs between wakefulness and sleep, so that it cannot be assumed with certainty that fetuses are in a stage comparable to unconsciousness (Derbyshire, 2010). Thus, interpretation of EEG activities is not sufficient to exclude that the welfare of the fetus can be compromised. So far, there is also no evidence that prenatal EEG measures are comparable with those of an adult. Furthermore, for human fetuses, it is consensus that potential for pain perception begins from week 22 to 23 with myelination of the spinal cord and brain; from week 26 onwards it is agreed that they can feel pain (Royal College of Obstetricians and Gynaecologists, 2010).

For bovine fetuses, the myelination of the nervous system begins already in week 20 (Maxie, 2015), and thus they potentially feel pain and uneasiness by week 22 . Additionally, humans are born in a relatively immature state (regarding mobility / motor function). In contrast, since cows are prey animals, newborn calves need to be able to follow the other animals of their flock in case they need to flee and therefore are born highly developed (i.e., relatively mature and mobile). A 2013 position paper ${ }^{1}$ from the Friedrich-Loeffler-Institute for Animal Welfare and Animal Husbandry reviewed the sentience of animal fetuses, starting a discussion about ethics and when the perception of pain or uneasiness begins. It concluded that severe suffering, or at least reduced welfare, possibly can be inflicted in the last trimester of the fetus.

\footnotetext{
1 https://www.amtstierarzt.de/fachthemen/download/10-tschg/64-2013-fli-stellungnahme-schlachtung-gravider-tiere
} 
Furthermore, when a pregnant animal is slaughtered, the fetus in the uterus shows movements that can last for several minutes until it dies of hypoxia. These movements can be an indicator for pain, even though the lung is immature. Overall, it is not proven whether fetuses experience lack of oxygen as pain. As long as this is uncertain, at least it should for ethical reasons be considered harmful to their well-being.

The information provided by Versteegen et al. (2021) inter alia "indicates that the fetus is not conscious, and therefore cannot feel pain (Mellor and Diesch, 2006)". Considering both this information and the information we provide above, we can conclude that there is conflicting information available and thus still no scientific consensus on the issue of whether bovine fetuses can suffer during and after slaughter of the dam. For that reason, these fetuses should be given the benefit of the doubt, analogous to the precautionary principle ${ }^{2}$ of the European Union.

\section{Transport of pregnant animals}

Next to the discussions on fetal suffering, it is unquestionable that the transport of pregnant animals can be a cause of increased animal suffering, at least for the dams. Therefore, restrictions on transporting pregnant animals have been implemented, e.g., for the last $10 \%$ of gestation in the European Union ${ }^{3}$. Some countries like Germany even forbid transport for pregnant cattle in their third trimester for the purpose of slaughter (albeit exceptions can be granted) ${ }^{4}$

The 2021 published White Paper "Live Animal Transport"5 on the revision of Council Regulation (EC) 1/2005 by Eurogroup for Animals even considers pregnant animals for whom $40 \%$ or more of the expected gestation period has already passed "unfit for transport". This implies that these animals "cannot be transport$e d$, regardless of the duration and quality of the journey". This obviously includes transport to slaughterhouses.

Beyond that, implementation of mandatory veterinary inspections of all female cattle (or at least mandatory performance of pregnancy testing) would detect most of the pregnant dams in free range herds or undocumented artificially inseminated cows in intensive farming. As the report of the European Food Safety Authority Panel on Animal Health and Welfare showed in their survey of 100 slaughterhouses from ten EU countries, there are multiple reasons for sending pregnant animals to slaughter, e.g., "poor record keeping on insemination and pregnancy checks" or "loss of respective information during trading" (AHAW et al., 2017). This report also dismisses the misconception of Versteegen et al. (2021) that it is "not economically viable to slaughter close-term dams since the calf is valuable", as it states that a "low calf price" is an economic reason for farmers to send pregnant cows to slaughter. For example, a calf of the breed German Holstein is sold for a price of only around 10 Euro (price retrieved on March 18, 2021 $)^{6}$. Hence, there would not be a financial incentive to keep the pregnant cow in such a case.

However, incentives for farmers to send pregnant cattle to slaughter exist, e.g., "anabolic effect of pregnancy (more muscles)"; "reducing risk of poor meat quality due to oestrus (dark cutting beef)"; "increased costs for keeping the cows" or even "calmer and more easily manageable cows" (AHAW et al., 2017).

The same report also estimates the median percentage of all mature female animals slaughtered in Europe while being pregnant as $16 \%$ for dairy cows and $11 \%$ for beef cattle. Of these pregnant animals, around 19\% (dairy cows) and around 14\% (beef cattle) are in the last third of gestation. This results in millions of slaughtered pregnant animals of which several hundred thousand are in their third trimester just in Europe alone.

On this matter, the statement of Versteegen et al. (2021) that not all countries have regulations in place that restrict transport and slaughter of pregnant animals strongly suggests that it is very likely that transportation of pregnant cattle happens far more often worldwide, since the overall majority of FBS is produced outside of Europe (93.5\%, according to an estimation by ISIA $)^{7}$.

Less strict standards in some countries cannot be an excuse to continue with business as usual when it goes against welfare of animals.

\section{Fetal blood collection}

It is a common misconception that the slaughter process is a "highly regulated area", as Versteegen et al. (2021) put it. Especially, extraction of fetal blood is far from being regulated or even transparent.

This is shown in their own quote of the OIE Terrestrial Animal Health Code, where they write "when fetal tissue is to be collected, the fetus should not be removed from the uterus until at least 15-20 minutes after the maternal neck or chest cut". However, in their quote, they leave out the two words "where practical" from the original OIE text ${ }^{8}$. Thus, the mentioned code is merely a recommendation on animal welfare protection.

Anyway, even when the time from the maternal neck or chest cut to the puncturing of the fetal heart is longer than 20 minutes, the fetus is still not being killed by a certified killing method.

For example, according to the German Regulations for Animal Protection and Slaughter (Tierschutz-Schlachtverordnung TierSchlV), if an animal is not killed by withdrawal of its blood, any further procedures on the animal may only be carried out af-

\footnotetext{
2 https://eur-lex.europa.eu/LexUriServ/LexUriServ.do?uri=CELEX:12008E191:EN:HTML

3 https://eur-lex.europa.eu/LexUriServ/LexUriServ.do?uri=CELEX:32005R0001:EN:HTML

${ }^{4}$ http://www.gesetze-im-internet.de/khfeverbg/_4.html

5 https://www.eurogroupforanimals.org/sites/eurogroup/files/2021-01/2020_01_27_efa_transport_white_paper_0.pdf

6 https://www.lksh.de/landwirtschaft/markt/rinder-und-kaelbernotierungen/

7 https://www.serumindustry.org/standardization/countries-of-origin/

8 https://www.oie.int/index.php?id=169\&L=0\&htmfile=chapitre_aw_slaughter.htm
} 
ter its confirmed death ${ }^{9}$. This means that simply waiting a certain amount of time would not be enough to fulfill animal welfare and legal regulations.

\section{Scientific issues with FBS use}

Finally, there is still the elephant in the room: The scientific issues every end-user faces (be it knowingly or unknowingly) when supplementing their cell and tissue culture media with FBS.

To name just a few:

- The undefined nature of FBS

- The variability between batches

- Possible contaminations

All these issues potentially inhibit reproducibility, interfere or distort experimental results, and cause increasing expenses due to purchasing and pre-testing of multiple batches (van der Valk et al., 2018). A continued use of FBS in the present and future can by no means solely be justified with discoveries from the past that involved FBS use as Versteegen et al. (2021) suggest. Furthermore, it cannot be proven that past discoveries would not have been made if FBS had not been available at that time. Instead, the abundant use of FBS in the last 60 years has delayed the search for and the definition of required elements in cell culture media.

Nevertheless, alternatives to FBS do exist and continue to prove their suitability ${ }^{10}$. Therefore, from an ethical point of view, there is no justification to continue using FBS any longer.

\section{Conclusion}

As long as there are no legally binding regulations that not only apply where practical, ethical and animal welfare issues remain immanent in the whole process of obtaining FBS. Existing regulations on transportation and slaughter of pregnant animals have to be followed and controlled, while being extended to at least meet the demands of Eurogroup for Animal's White Paper ${ }^{5}$. Furthermore, a consistent legal framework to monitor and record (artificially induced and naturally occurring) pregnancies in bovines as well as a veterinary examination (or a mandatory pregnancy test) before transport, has to be implemented. This would ensure that pregnant dams and their fetuses do not end up at slaughterhouses in the first place and therefore drastically reduce the number of fetuses used in ethically questionable blood collection procedures. Hence, fetal blood should not be simply deemed to be a "byproduct" of the meat and dairy industry forever.

In the meantime, while FBS is still being produced, legally binding regulations are indispensable and must at least implement mandatory anesthesia and/or humane killing of the fetuses (Weber and Wagner, 2021). There are good reasons to claim that they do experience some kind of pain during the procedure. Thus, the collection of fetal blood is strongly linked to suffering, which underlines the urgency of animal welfare improvements concerning this topic. And as long as animals are still used for human purposes, maximizing their welfare should be an ethical necessity, not maximizing their potential in the (human-centered) food chain.

Implementing animal welfare standards will disrupt the most unethical practices of the current system and bring it closer to a balance between animal and human needs that is not built on suffering and distress.

We reconfirm our invitation to the serum-producing industry to encourage discussions to find a common solution for the animal welfare issues and the development of animal-friendly in vitro media supplements, not only for ethical reasons but also to contribute significantly to safe, reproducible and consistent advances in medical and veterinary health.

\section{References}

AHAW - EFSA Panel on Animal Health and Welfare, More, S., Bicout, D. et al. (2017). Animal welfare aspects in respect of the slaughter or killing of pregnant livestock animals (cattle, pigs, sheep, goats, horses). EFSA J 15, e04782. doi:10.2903/j. efsa.2017.4782

Bellieni, C. V. (2019). New insights into fetal pain. Semin Fetal Neonatal Med 24, 101001. doi:10.1016/j.siny.2019.04.001

Derbyshire, S. W. (2010). Foetal pain? Best Pract Res Clin Obstet Gynaecol 24, 647-655. doi:10.1016/j.bpobgyn.2010.02.013

Gstraunthaler, G., Lindl, T. and van der Valk, J. (2013). A plea to reduce or replace fetal bovine serum in cell culture media. Cytotechnology 65, 791-793. doi:10.1007/s10616-013-9633-8

Hodgson, J. (1991). Checking sources: The serum supply secret. Biotechnology 9, 1321-1324. doi:10.1038/nbt1291-1320

Jochems, C. E., van der Valk, J. B., Stafleu, F. R. et al. (2002). The use of fetal bovine serum: Ethical or scientific problem? Altern Lab Anim 30, 219-227. doi:10.1177/026119290203000208

Maxie, G. (2015). Jubb, Kennedy \& Palmer's Pathology of Domestic Animals: 3-Volume Set (338). $6^{\text {th }}$ edition. Elsevier Health Sciences. ISBN: 9780702053221

Mellor, D. J. and Diesch, T. J. (2006). Onset of sentience: The potential for suffering in fetal and newborn farm animals. Appl Anim Behav Sci 100, 48-57. doi:10.1016/j.applanim.2006.04.012

Royal College of Obstetricians and Gynaecologists (2010). Fetal Awareness: ReviewofResearchandRecommendationsfor Practice. London, UK: RCOG Press. https://www.rcog.org.uk/globalassets/ documents/guidelines/rcogfetalawarenesswpr0610.pdf

van der Valk, J., Bieback, K., Buta, C. et al. (2018). Fetal bovine serum (FBS): Past - Present - Future. ALTEX 35, 99-118. doi:10. 14573/altex.1705101

Versteegen, R., Murray, J. and Doelger, S. (2021). Animal welfare and ethics in the collection of fetal blood for the production of fetal bovine serum. ALTEX 38, 319-323. doi:10.14573/ altex. 2101271

Weber, T. and Wagner, K. (2021). Replacing fetal bovine serum (FBS) in research and testing. ALTEX 38, 163-164. doi:10. 14573/altex.2012141

9 https://www.gesetze-im-internet.de/tierschlv_2013/_12.html

10 https://fcs-free.org/ 